\title{
LAS GUERRAS DEL HUMMUS: COMIDA LOCAL, RÉCORD GUINNESS Y GASTROPOLÍTICA PALESTINO-ISRAELÍ1
}

\author{
NIR AVIELI \\ Universidad Ben Gurión
}

En mayo de 2008, un grupo de cocineros israelíes elaboró un platillo de bummus de 400 kilogramos y estableció así el récord mundial Guinness. La proeza desató lo que luego se conoció como las "guerras del bummus", 2 es decir, acometidas recurrentes de competidores israelíes y libaneses para establecer nuevos récord Guinness del platillo de hummus más grande del mundo. Estos sucesos culinarios fueron discutidos por participantes, espectadores y medios de comunicación de Israel y Líbano en términos nacionalistas para implicar que los intentos por romper el récord Guinness involucraban pretensiones de propiedad nacional. Los acontecimientos dieron un giro inesperado cuando un restaurantero palestino-israelí de la villa de Abu Gosh, cerca de Jerusalén, decidió establecer un nuevo récord para Israel. Todo esto atrajo gran atención pública y mediática en Israel y más allá, lo que provocó enigmáticas cuestiones sobre el papel de los alimentos en las políticas de identidad nacional en zonas de conflicto en general y en las zonas fronterizas palestino-israelíes en particular.

Los antropólogos están poniendo creciente atención a la "comida local" $\mathrm{y}$ los procesos culturales que la definen como

Este artículo fue recibido por la dirección de la revista el 17 de abril de 2013 y aceptado para su publicación el 16 de julio de 2013.

${ }^{1}$ Deseo agradecer a Rafi Grosglik su valioso apoyo en el desarrollo de las ideas presentadas en este artículo. 42 .

${ }^{2}$ A. Ariel, “The Hummus Wars”, Gastronomica, vol. 12, núm. 1, 2012, pp. 34-

${ }^{3}$ M. Ashkenazi y J. Jacob, The Essence of Japanese Cuisine: An Essay on Food and Culture, Richmond, Curzon, 2000; C. M. Counihan, Around the Tuscan Table: Food, Family and Gender in Twentieth-Century Florence, Nueva York, Routledge, 2004; 
tal, lo que muestra una y otra vez cómo la idea de "nuestra comida" es construida mediante procesos de negociación y adaptación constantes. Las guerras del hummus, sin embargo, son diferentes en el sentido de que el debate no es sobre la preparación adecuada, las recetas auténticas, la pertenencia a la ecología o el terruño locales, ni sobre la calidad; en vez de eso, se refieren a cuestiones directas de propiedad y poder, traducidas en la pregunta machista cuantificable: ¿Cuál bummus es más grande?

La singularidad de las guerras del bummus se exacerba por el hecho de que este antiguo platillo mundano es reivindicado por Estados nacionales modernos relativamente jóvenes, enfrascados en un conflicto militar activo. Así pues, mientras la mayor parte del trabajo académico citado arriba destaca la distinción, ${ }^{4}$ el orgullo nacional y la pureza cultural, las guerras del bummus disputan la apropiación de la comida del otro o, más precisamente, la apropiación de la comida del enemigo. ${ }^{5}$ Más importante: estos acontecimientos son enigmáticos debido al inesperado papel protagónico de mediadores, asumido por palestinos con ciudadanía israelí.

En este artículo, basado en un proyecto etnográfico de largo plazo sobre los hábitos alimentarios israelíes, realizado en varios lugares, muestro cómo las partes involucradas en el conflicto culinario asignaron al bummus significados que entretejen historias e imágenes de la región y las lealtades políticas. Específicamente, abordo los significados atribuidos por palestino-israelíes al platillo y, a través de él, a sí mismos. En la

N. Avieli, "Vietnamese New Year Rice Cakes: Iconic Festive Dishes and Contested National Identity", Ethnology, vol. 44, núm. 2, 2005, pp. 167-187; R. Wilk, Home Cooking in the Global Village: Caribbean Food from Buccaneers to Ecotourists, Londres, Berg, 2006; N. Klumbyte, "The Soviet Sausage Renaissance”, American Anthropologist, vol. 112, núm. 1, 2010, pp. 22-37; J. L. Watson (ed.), Golden Arches East: McDonald's in East Asia, Stanford, Stanford University Press, 1997, pp. 183-200; M. Caldwell, "Domesticating the French Fry: McDonald's and Consumerism in Moscow", Journal of Consumer Culture, vol. 4, núm. 1, 2004, pp. 5-26; N. Avieli, "Roasted Pigs and Bao Dumplings: Festive Food and Imagined Transnational Identity in Chinese-Vietnamese Festivals", Asia Pacific Vieropoint, vol. 46, núm. 3, 2005, pp. 281-293.

${ }^{4}$ P. Bourdieu, Distinction: A Social Critique of the Judgment of Taste, Cambridge, Harvard University Press, 1984.

${ }^{5}$ D. Hirsch, "Hummus is Best when it is Fresh and made by Arabs: The Gourmetization of Hummus in Israel and the Return of the Repressed Arab", American Ethnologist, vol. 38, núm. 4, 2011, pp. 617-630. 
conclusión regreso a la sustancia en sí misma y sugiero que las cualidades específicas del bummus (platillo mundano y público hecho de diversas maneras con recetas diferentes y que a menudo es compartido entre comensales) lo hacen excepcionalmente adecuado para la expresión de ideas complejas, e incluso opuestas, relacionadas con las identidades colectivas.

\section{Comer bummus en Israel}

Hummus es la palabra árabe que designa tanto los granos de garbanzo como el puré de garbanzo. ${ }^{6}$ El puré se hace con garbanzos hervidos que luego son amasados o molidos y después mezclados con jugo de limón, tahini (pasta de ajonjolí) y aceite de oliva. Se sazona con ajo, sal y pimienta, pero hay muchas variaciones e ingredientes adicionales, así como recetas secretas. Puede ser servido con una pizca de aceite de oliva y una cucharada de salsa tabini (pasta de ajonjolí con limón y agua), con garbanzos enteros o amasados y algo de páprika (pimentón) o perejil, que añaden sabor y color. Msabaha es un platillo del desayuno palestino (sabab significa mañana en árabe) hecho de granos de hummus hervidos (no molidos), con tabini y aceite de oliva, servido con una salsa picante de chiles encurtidos y limón. Otra variante incluye pasta foul (haba cochinera) y huevos cocidos duros.

En los restaurantes palestinos de hummus en Israel, el puré de garbanzo en sus diversas formas puede ser servido en un tazón como platillo único, acompañado de pan pita y un plato pequeño de aceitunas, cebolla, tomate y pepinos encurtidos. Los comensales arrancan trozos de pan, los sumergen en el tazón y recogen una porción de bummus que se llevan a la boca. Esta porción (usualmente servida con dos piezas de pan) equivale a un desayuno sustancioso para una persona, pero puede ser suficiente para dos y a menudo es compartida.

Por su alto contenido de proteína vegetal, grasas no saturadas y fibra, complementado con carbohidratos (pita), minerales

${ }^{6}$ El término bíblico himtza es el correcto para referirse a los granos de garbanzo en hebreo, pero en el hebreo moderno casi no se usa, mientras que hummus es el término común para los granos y el platillo. 
y vitaminas (vegetales frescos y encurtidos), el bummus es un alimento completo, nutritivo, barato y altamente satisfactorio. El bummus es también un platillo urbano que se elabora en cocinas especializadas en las ciudades y pueblos del Medio Oriente. Como el falafel, el hummus es un alimento tradicional de la clase obrera árabe (no de los granjeros). El hecho de que sea un platillo urbano moderno y no un platillo tradicional que se consuma en contextos marginales contribuye a su adecuación como acompañante de la comunicación de ideas sobre asuntos sociales contemporáneos.

Sin embargo, el bummus puede ser consumido también en ambientes sofisticados. En restaurantes de categoría es servido en platos pequeños, acompañado de otros tipos de "ensaladas" o pastas como tabini, labane (queso suave de cabra), baba ganoush (puré de berenjena asada), matbouba (salsa de tomate condimentada), taboule (ensalada de trigo bulgur), aceitunas, pepinillos, etcétera, al estilo mediterráneo meze. Un plato pequeño de bummus, junto con otras pastas y ensaladas, puede ser servido también como entrada de banquetes de carnes asadas y platillos cocinados. En estos casos, los comensales también acostumbran sumergir trozos de pita o de carne en un tazón común para recoger hummus. La comensalidad es pues un atributo común del bummus en los ambientes culinarios tanto mundanos como sofisticados; en efecto, la compartición es un componente importante de la experiencia del bummus y también un componente importante del argumento del presente artículo.

Dafna Hirsch, ${ }^{7}$ en su estudio de la historia del bummus en Israel, señala que los sionistas pioneros - judíos de Europa oriental que migraron a Palestina a principios del siglo xx para crear el Estado judío- incorporaron muy pronto el bummus a su repertorio culinario como parte de sus intentos prácticos y simbólicos de establecerse en la región. Los judíos de los países del Medio Oriente que migraron a Israel después de la creación del Estado, en 1948, y que estaban familiarizados con el platillo, aumentaron su demanda. Los empresarios judío-israelíes industrializaron y modernizaron la producción de bummus, primero como conserva y después como "ensalada" fresca 
refrigerada. En años recientes, industriales judío-israelíes han globalizado el bummus con fábricas en Europa y Norteamérica. Una estrategia de mercadeo en Israel es la reciente diversificación y "gourmetización" del hummus por grandes fábricas y productores de "boutique". Otra tendencia reciente es la producción de bummus orgánico. ${ }^{8}$

Es importante notar que el bummus es muy popular entre los judío-israelíes. Los establecimientos culinarios que ofrecen bummus en sus diversas formas son los negocios de comida rápida y barata, más comunes en el país, mientras las encuestas muestran constantemente que el bummus es el alimento más común en los refrigeradores de los hogares israelíes. Por consiguiente, los judío-israelíes consideran el bummus como parte estable de su cultura culinaria. También es importante tener en mente que, aunque algunos establecimientos palestino-israelíes y judíos sirven bummus hecho en casa, la mayoría de los restaurantes judíos, y muchos palestinos, sirven bummus industrializado. En este sentido, el bummus es similar a muchos otros "alimentos locales" que ostentan una imagen de autenticidad, aunque sean industrializados y producidos en masa.

\section{Comida local e identidad local}

Es un axioma antropológico que los alimentos son una poderosa señal de identidad. " "El alimento - sostiene Fischler-es central para la identidad individual en [la medida en] que todo individuo humano está biológica, psicológica y socialmente construido por los alimentos que él/ella elige incorporar"; ${ }^{10}$ mientras que Sutton asevera que "el alimento se refiere a creación y mantenimiento de la identidad, ya sea nacional, étnica, de clase o de género". ${ }^{11}$ Los antropólogos han mostrado

${ }^{8}$ R. Grosglik, "Organic Hummus in Israel: Global and Local Ingredients and Images”, Sociological Research Online, vol. 16, núm. 3, 2011.

${ }^{9}$ Wilk, Home Cooking in the Global Village, op. cit.

${ }^{10} \mathrm{C}$. Fischler, "Food, Self and Identity", Social Science Information/sur les sciences sociales, vol. 27, núm. 2, 1988, pp. 275-292.

${ }^{11}$ D. E. Sutton, Remembrance of Repasts: An Anthropology of Food and Memory, Oxford, Berg, 2001, p. 5. 
repetidamente cómo los hábitos alimentarios comunes y los gustos compartidos crean las bases de la membresía de grupo, ${ }^{12}$ mientras que los tabús y los estereotipos de alimentos establecen límites sociales mediante la definición de los alimentos de otros como contaminantes e incomestibles. ${ }^{13}$

El alimento, sostiene Fischler, ${ }^{14}$ deviene epítome de identidad mediante el proceso de incorporación, en el que la materia comestible, culturalmente transformada, cruza los límites corporales y rompe las dicotomías adentro-afuera y mundo-yo. El famoso aforismo de Brillat Savarine, "Dime qué comes y te diré quién eres”, ejemplifica este proceso, pues sugiere que cuando comemos nos volvemos perfectos consumidores de nuestra cultura al internalizar físicamente sus principios y valores, ingiriéndolos y digiriéndolos en nuestros cuerpos.

No obstante, el alimento no es un símbolo simple y estático de identidad colectiva. La cocina evoluciona en contextos históricos y sociales específicos, ${ }^{15} \mathrm{y}$ hace de los platillos y hábitos alimentarios representaciones materiales de narrativas históricas particulares y arreglos sociales singulares. ${ }^{16}$

${ }^{12}$ R. Wilk, “'Real Belizean Food': Building Local Identity in the Transnational Caribbean”, American Antbropologist, vol. 101, núm. 2, 1999, pp. 244-255; G. Holt y V. Amilien, "Introduction: From Local Food to Localized Food", Anthropology of Food, número especial sobre productos y sistemas agroalimentarios locales, 2007.

${ }^{13}$ M. Douglas, Implicit Meanings: Selected Essays in Anthropology, Hove, Psychology Press, 1975; J. Bahloul, "Food Practices among Sephardic Immigrants in Contemporary France: Dietary Laws in Urban Society", Journal of the American Academy of Religion, vol. 63, núm. 3, 1995, pp. 485-496. Un ejemplo sorprendente de las fuertes asociaciones que la gente hace entre los alimentos que come y su sentido de identidad colectiva es evocado por DeSoucey en un artículo reciente (M. DeSoucey, "Gastronationalism Food Traditions and Authenticity Politics in the European Union”, American Sociological Review, vol. 75, núm. 3, 2010, pp. 432-455), donde relata que una "informante potencial, una profesora" (ibid., p. 446) aceptó ser entrevistada sobre el foie gras sólo si DeSoucey misma “disfrutaba comer foie gras". La entrevistada explicó después que debido a que algunos estadounidenses estaban contra la producción de foie gras, ella se reuniría con la entrevistadora sólo si le gustaba el foie gras, pues "ésta [era] una cuestión de solidaridad nacional” (idem.).

${ }^{14}$ Fischler, "Food, Self and Identity", op. cit.

${ }^{15}$ Klumbyte, “The Soviet Sausage Renaissance”, op. cit.; Watson, Golden Arches East, op. cit.; S. Mintz, Sweetness and Power: The Place of Sugar in Modern History, Nueva York, Viking, 1986. J. Goody, Cooking, Cuisine and Class: A Study in Comparative Sociology, Cambridge, Cambridge University Press, 1985; S. Mennell, All Manners of Food: Eating and Taste in England and France from the Middle Ages to the Present, Oxford, Blackwell, 1985; Wilk, Home Cooking in the Global Village, op. cit.

${ }^{16}$ N. Ries, "Potato Ontology: Surviving Postsocialism in Russia", Cultural 
En la literatura especializada hay una interesante división entre los estudios sobre la identidad inmersa en los alimentos exóticos de los pueblos marginales, los estudios sobre hábitos alimentarios postsocialistas y los estudios sobre las preparaciones culinarias occidentales. Cuando se trata de los alimentos consumidos por pueblos exóticos - objetos tradicionales de la antropología-, la atención se fija principalmente en las maneras en que platillos específicos y modos de consumirlos representan facetas de la identidad nacional o de la identidad étnica. ${ }^{17}$ En el contexto postsocialista, el tema a la mano es a menudo la modernización de la producción de alimentos específicos. ${ }^{18}$ Pero en el estudio de los hábitos alimentarios occidentales, la atención se concentra sobre todo en los debates gubernamentales e institucionales sobre los derechos de marcas comerciales de lucrativos alimentos específicos en regiones particulares, principalmente de Estados Unidos. ${ }^{19}$ Probablemente esto es resultado de las estructuras de poder académico prevalecientes, basadas en dicotomías imaginadas entre tradición y modernidad y entre racionalidad y emociones.

El caso del bummus en Israel desafía y complica estas distinciones. Aunque es claramente un platillo palestino, el bummus es considerado por muchos israelíes como el platillo nacional de su propio Estado-nación. Y aunque es un platillo de la clase

\footnotetext{
Anthropology, vol. 24, núm. 2, 2009, pp. 181-212; K. O’Connor, “The Hawaiian Luau: Food as Tradition, Transgression, Transformation and Travel", Food, Culture and Society, vol. 11, núm. 2, 2008, pp. 149-172.

${ }^{17}$ Ashkenazi y Jacob, The Essence of Japanese Cuisine, op. cit.; U. Narayan, Dislocating Cultures: Identities, Traditions and Third-World Feminism, Nueva York-Londres, Routledge, 1997; Jeffrey M. Pilcher, ;Que vivan los tamales!: Food and the Making of Mexican Identity, Albuquerque, University of New Mexico Press, 1998; S. Howell, "Modernizing Mansaf: The Consuming Contexts of Jordan's National Dish", Food and Foodways, vol. 11, núm. 4, 2003, pp. 215-243; I. Cusack, "African Cuisines: Recipes for Nation Building?”, Journal of African Cultural Studies, vol. 13, núm. 2, 2000, pp. 207-225; K. J. Cwiertka, Modern Japanese Cuisine: Food, Power and National Identity, Londres, Reaktion Books, 2006.

${ }^{18}$ Klumbyte, "The Soviet Sausage Renaissance", op. cit.; Ries, "Potato Ontology", op. cit.

${ }^{19}$ M. Winter, "Embeddedness, the New Food Economy and Defensive Localism", Journal of Rural Studies, vol. 19, núm. 1, 2003, pp. 23-32; J. Cavanaugh, "Making Salami, producing Bergamo: The Transformation of Value", Ethnos, vol. 72, núm. 2, 2007, pp. 149-172; DeSoucey, "Gastronationalism Food Traditions...", op. cit.; G. Welz, "Contested Origins: Food Heritage and the European Union's Quality Label Program”, Food, Culture and Society, vol. 16, núm. 2, 2013, pp. 265-279.
} 
obrera, servido rutinariamente en tazones colectivos en los que se introducen los dedos para sumergir el pan pita en la pasta, gran parte del hummus del Medio Oriente es producido hoy en día en fábricas modernas y es exportado como alimento saludable, sofisticado, por distribuidores multinacionales como Uniliver y Nestlé a los llamados mercados "occidentales” y "desarrollados". ${ }^{20}$

En las siguientes páginas describo la evolución de las guerras del hummus y luego me aboco a la etnografía detallada de uno de sus principales sucesos: el establecimiento del récord Guinness del platillo de bummus más grande del mundo en la villa palestino-israelí de Abu Gosh. En la discusión regreso a la literatura antropológica que trata directamente el significado del hummus en el Israel contemporáneo y sugiero que el análisis de las guerras del bummus permite una lectura sensible y matizada de las relaciones judío-árabes en Israel.

\section{Las guerras del hummus}

En mayo de 2008, un grupo de cocineros israelíes elaboró un plato de bummus de unos 400 kilogramos, y estableció así el récord mundial Guinness. ${ }^{21} \mathrm{La}$ actividad fue patrocinada por el productor de ensaladas israelí Sabra, miembro del grupo Osem, uno de los productores líderes de alimentos de Israel, propiedad de la megaempresa de alimentos Nestlé. El reto tuvo un claro contorno económico y probablemente fue parte de la campaña de mercadeo de las ensaladas Tzabar de Nestlé en la Unión Europea y Estados Unidos.

El nombre de la marca, sin embargo, apunta a temas de identidad nacional y étnica: sabra (en yiddish; tzabar en hebreo) o tuna de nopal, es usado en hebreo moderno para denotar también a los judíos nacidos en Israel, como los judío-israelíes tienden a describirse a sí mismos, espinosos por fuera pero dulces y suaves por dentro. ${ }^{22}$ Paradójicamente, el cactus sabra

${ }^{20} \mathrm{John}$ T. Edge, "Hummus catches on in America (as Long as It's Flavored)", The New York Times, 15 de junio de 2010.

${ }^{21}$ Itamar Eichner, “The 2nd Hummus War”, Ynetneres, 28 de enero de 2010.

${ }^{22}$ O. Almog, Ha-tzabar-dyokan, Tel Aviv, Am Oved, 1997. 
es en realidad una planta del Nuevo Mundo de reciente arribo, resistente a la sequía, que fue usada por los agricultores palestinos para delimitar sus campos. Como consecuencia, el cactus crece hoy en día en las ruinas de las villas palestinas. ${ }^{23}$ Este símbolo culinario es por ello multivocal y evoca tanto una autopercepción afirmativa de los judío-israelíes como la memoria de los palestinos desplazados.

El récord Guinness 2008 para el platillo de bummus más grande del mundo no pasó desapercibido. En octubre de ese año, Fadi Abboud, presidente de la Asociación de Industriales Libaneses y después ministro de turismo de Líbano, anunció que su país demandaría a Israel por violar sus derechos de propiedad culinarios: "[Al] comercializar platillos nacionales libaneses como el bummus y el tabboule ${ }^{24}$ como propios [...] Israel estaba haciendo perder decenas de millones de dólares por año a Líbano". ${ }^{25}$ Abboud explicó que la demanda de Líbano se basaría en el "precedente del queso feta, por el cual una corte europea reconoció a Grecia el derecho exclusivo a usar la palabra feta para el queso que producía". ${ }^{26}$

$\mathrm{Al}$ parecer, los libaneses no encontraron el tribunal competente, pues la demanda nunca fue presentada; sin embargo, el asunto fue significativo no sólo económica, sino también simbólicamente, y estuvo muy enmarañado con percepciones de identidad y orgullo nacionales. En consecuencia, Líbano decidió responder al desafío israelí en octubre de 2009, y estableció su propio récord Guinness con un plato que contenía no menos de 2000 kilogramos de bummus. ${ }^{27}$

La respuesta israelí a este nuevo récord fue rápida; en noviembre del mismo año, 80 kilogramos de carne y vísceras de pollo, 12 kilogramos de ensalada y 10 kilogramos de harina fueron cocinados para rellenar un gigantesco pan pita de tres

${ }^{23}$ E. Ben-Ze'ev, Remembering Palestine in 1948: Beyond National Narratives, Cambridge, Cambridge University Press, 2011.

${ }^{24}$ Ensalada árabe del Medio Oriente tradicionalmente hecha de trigo bulgur, tomate, perejil, cebolla, aceite de oliva y jugo de limón.

${ }^{25}$ Shuki Galili, “The Third Lebanon War: The Fight over Hummus”, Yediot Ahronot, 2 de noviembre de 2009; G. Lichfield, "Food Fight: A Look Inside the Middle East's New Weapons of Mass Consumption”, Foreign Policy, 15 de enero de 2010.

${ }^{26} \mathrm{Idem}$.

${ }^{27}$ Ariel, "The Hummus Wars", op. cit. 
metros de diámetro a fin de imponer el récord Guinness para el Meorav Yerushalmi (asado Jerusalén): platillo de carne y vísceras de pollo asadas con cebolla y sazonadas con cúrcuma, ajo y comino, servido en pan pita con ensalada y tabini. Este platillo, reputadamente inventado en la década de 1970 en el mercado municipal Mahane Yehuda, de Jerusalén, es un "platillo de sobras" icónico y una especialidad popular local. El mercado municipal Mahane Yehuda es también un centro de nacionalismo israelí, política derechista y sentimientos antiárabes-palestinos, así que la reacción y el lugar hacen sentido en términos israelíes.

Aunque el asado Jerusalén no es bummus, los reportes de los medios de comunicación israelíes dejaron claro que eso era otra maniobra de las guerras del hummus. El Canal 2 de Noticias, principal emisor de noticias del país, empezó su reporte de la siguiente forma: "Mientras los libaneses 'robaban' [comillas en el texto original del sitio web adyacente al video] nuestro récord Guinness del platillo de bummus más grande del mundo, Israel decidió contraatacar (lehashiv milhama) con una proeza que los vecinos norteños difícilmente podrían igualar: el asado Jerusalén más grande del mundo". ${ }^{28}$ Los encabezados del popular sitio web de noticias Walla fueron más directos aún: "Otra victoria para Israel: récord en asado Jerusalén". ${ }^{29}$

Sin embargo, la futilidad de la táctica fue advertida por un reportero de $A l$ Arabiya (sitio en internet de noticias), que escribió: "no debieron haber tenido problemas para conseguir su mixtura de 200 kilogramos (440 libras) de vísceras de pollo reconocida por el libro de récords Guinness: nadie lo había intentado antes". ${ }^{30}$

En efecto, el récord Guinness por el asado Jerusalén más grande no satisfizo al lado israelí. En un giro sorprendente, Ibrahim Jawadat, palestino-israelí ${ }^{31}$ de la villa de Abu Gosh y

${ }^{28}$ Ilanit Adler, "Watch: The World Largest Jerusalem Mix", Channel 2 Newes, 30 de noviembre de 2009.

${ }^{29}$ Walla Editors, “Another Victory for Israel: Jerusalem Mix Record”, Walla! Neres, 30 de noviembre de 2009.

${ }^{30}$ Occupied Jerusalem (AFP), “Israelis Seek World Record With 'Jerusalem Mix'”, Al Arabiya Newes, 30 de noviembre de 2009.

${ }^{31}$ Los palestinos que permanecieron en el territorio del nuevo Estado de Israel, creado después de la guerra de 1948, recibieron la ciudadanía israelí. Un término más 
propietario del popular restaurante Hummus Abu Gosh, anunció que al duplicar el récord libanés y preparar un platillo con no menos de 400 kilogramos de hummus, "estamos reparando el honor estatal". ${ }^{32}$

Abu Gosh es un lugar interesante ahora. La villa ostenta docenas de restaurantes especializados en comida palestina como falafel, tabini, taboule y, sobre todo, hummus. Estos establecimientos atienden principalmente a clientes judío-israelíes, es decir, gente que figura entre la más próspera.

Aunque muchas villas palestino-israelíes tienen restaurantes similares, $\mathrm{Abu}$ Gosh es excepcionalmente popular entre los judío-israelíes porque sus residentes son considerados "árabes buenos". Son conocidos así por dos razones. La primera es histórica: en 1948, el jefe de la villa (mukhtar) hizo un trato con los líderes de la Hagana (organización de defensa del preestado judío) en el sentido de que la villa permanecería neutral en el conflicto armado a cambio de protección judía. La parte menos conocida de la historia es que, pese a haber permanecido neutrales e incluso ser apoyados por los judíos, muchos residentes de Abu Gosh fueron deportados junto con moradores de villas palestinas vecinas al terminar la guerra, y sólo se les permitió regresar gracias a una campaña civil encabezada por prominentes figuras judías.

En su etnografía de Abu Gosh, Rebeca Stein ${ }^{33}$ cita a varios residentes que cuentan amargos recuerdos de sus relaciones con los judíos y subrayan el hecho de que, pese a su colaboración con ellos, la mayoría de las tierras de las villas les fueron confiscadas. No obstante, los informantes manifestaron su deseo de dejar a un lado esos sentimientos negativos con tal de preservar la buena imagen de la villa como un lugar amistoso y seguro para los judíos.

La segunda razón de la popularidad de Abu Gosh entre los judío-israelíes tiene que ver con la creencia generalizada de que

\footnotetext{
adecuado para ellos sería "Palestinos con ciudadanía israelí", pero yo opto por el término comúnmente usado por judíos y palestinos.

${ }^{32}$ Didi Harari, "Owner of Abu Gosh Restaurant to break the Lebanese Guinness record” (entrevista con Ibrahim Jawadat), 103FM, 8 de noviembre de 2009.

${ }^{33}$ R. Stein, Itineraries of Conflict; Israeli, Palestinians and the Political Lives of Tourism, Durham, Duke University Press, 2003.
} 
Abu Gosh es una villa cristiana y, como tal, segura, o al menos más segura para los judíos en comparación con las villas musulmanas. La imagen cristiana de Abu Gosh está construida en torno de su iglesia de Notre Dame, el gran monasterio benedictino y especialmente el muy popular Festival de Música Litúrgica de Abu Gosh. Sin embargo, la Oficina Central de Estadísticas indica que $99.9 \%$ de los residentes de la villa son musulmanes. ${ }^{34}$

La popularidad de Abu Gosh como la "meca del bummus" de Israel se basa, pues, en un conjunto de supuestos erróneos de judío-israelíes respecto de la religión de sus residentes y de sus relaciones con el Estado de Israel y con los ciudadanos judíos.

Ibrahim Jawadat mismo es una persona interesante. Según reportes periodísticos salió de Israel muy joven, posiblemente por deudas financieras, para reunirse con su hermano en Estados Unidos, pero después de ganar millones de dólares en la lotería de Illinois regresó a Israel, donde puso el restaurante Hummus Abu Gosh, así como otros negocios, y se estableció como un empresario exitoso. ${ }^{35}$ Hablamos pues de un musulmán palestino de una villa que los judío-israelíes tienen por cristiana y amistosa con ellos, la cual proclama que reparará el honor nacional temporalmente perdido en las guerras del bummus con Líbano.

Naturalmente, encontré muy interesante la cadena de acontecimientos y enfilé hacia Abu Gosh con mi colega Rafi Gorsglik, el 8 de enero de 2010, a fin de atestiguar el intento de establecer un nuevo récord Guinness para el platillo de bummus más grande del mundo.

En efecto, la dinámica de los sucesos resultó inesperada. Mientras la retórica mediática (una parte de la cual escuché hasta la saciedad en la radio mientras conducía hacia Abu Gosh) era "nacional" e incluso nacionalista, expresada en términos tales como "nosotros", "los libaneses" y "nuestro platillo nacional”, la atmósfera en Abu Gosh era totalmente diferente. Para empezar, la villa estaba decorada con sus propias banderas municipales, no con banderas israelíes. Lo "local” era también

${ }^{34}$ Local Councils in Israel, "Profile of Local Councils 2009”, publicación 1451.

${ }^{35}$ Tani Goldstin, "Jawadath Ibrahim: Abu Gosh is not only Hummus", Yediot Abronot, 20 de enero de 2006. 
el tema principal de los discursos, los cuales destacaban el papel de los palestinos ciudadanos de Israel como mediadores potenciales entre los ciudadanos judíos de Israel y sus vecinos árabes.

Un rasgo importante e inusual del suceso fue su bilingüismo. El hebreo, el árabe y el inglés son las lenguas oficiales del Estado de Israel, pero el árabe suele ser desaprobado y evitado en actividades no exclusivamente árabes. Aquí los anfitriones decidieron usar ambas lenguas. El acto fue encabezado por Zuhair Bahloul, periodista palestino-israelí que a menudo es ridiculizado por los judío-israelíes debido a su hebreo docto y su marcado acento árabe. El "Ídolo de Israel", la palestina israelí Miriam Toukan, cantó en árabe y en hebreo la canción Aleluya, ganadora en Eurovisión.

El discurso bilingüe de Ibrahim Jawadat destacó la "abugoshnidad" del acto; repetidamente subrayó que se estaba realizando en Abu Gosh, sin mencionar el Estado de Israel, como tampoco lo había hecho en entrevistas con los medios nacionales controlados por judíos. Subrayó también que el bummus es una especialidad tradicional local (es decir, platillo palestino, no israelí). Pero su argumento principal fue que "los árabesisraelíes son el puente para la paz en el Medio Oriente". Refirió que líderes judíos, árabes y occidentales como Yitzhak Rabin, Shimon Peres, Jimmy Carter y Anwar Sadat ${ }^{36}$ se reunieron en Abu Gosh y negociaron la paz frente a un plato de bummus, y llamó a tener más reuniones y pláticas de paz entre líderes judíos y árabes en $\mathrm{Abu}$ Gosh.

Por extraño que parezca, el 8 de enero de 2010 fue el día más caluroso recordado en un invierno israelí, con temperaturas de hasta 30 grados Celsius. Ya que enero es normalmente frío y nuboso, no se tomaron precauciones contra el calor en términos de sombra y refrigeración.

Llegamos a Abu Gosh temprano, justo antes de la llegada de un camión grande con la etiqueta "Salately Mickey" ("En-

${ }^{36}$ Mencionó también a Lord Bernadotte como uno de los negociadores de paz frente a un plato de hummus en Abu Gosh. Diplomático sueco, Bernadotte fue el mediador del Consejo de Seguridad de Naciones Unidas en el conflicto árabe-israelí de 1947-1948; fue asesinado en 1948 por el grupo militante sionista Lehi ("Combatientes por la libertad de Israel”). Es inconcebible que Bernadotte comiera bummus en Abu Gosh. 
saladas Mickey”, socios comerciales judíos de Jawadat). Enseguida se descargaron del camión cajas de bummus comercial prefabricado y su contenido fue vertido en una enorme antena parabólica prestada por el vecino centro satelital Neve Ilan. La antena fue colocada sobre una pesa ubicada en una rampa por encima del estacionamiento del restaurante. De pie junto a los trabajadores, no pudimos dejar de notar el olor acre del bummus, que parecía echado a perder.

Conforme el día avanzó y la temperatura aumentó, pensábamos en el hummus expuesto al tremendo sol y al creciente calor. Nos imaginamos que había empezado a agriarse y que su delicada pasta comenzaba a ponerse putrefacta. El acceso a la rampa del escenario estaba cerrado y no pude evaluar el estado del hummus. Sin embargo, pese a que se había prometido que se distribuiría gratis entre los espectadores una vez que se estableciera el récord (promesa que atrajo a muchos, según sus propios testimonios medio en broma), no fue así. No hubo explicación alguna de los organizadores ni reportes en medios de comunicación después.

Docenas de hombres con mandiles blancos y gorros de chef se movían entre la multitud. Cuando pregunté a algunos de ellos qué estaban haciendo, me explicaron que en realidad eran camareros de algunos restaurantes locales, invitados por los organizadores a caminar por el escenario con uniformes de cocina. A pregunta nuestra admitieron no haber preparado el bummus. Identificamos a algunos de ellos como participantes en la construcción del escenario; supusimos que habrían cambiado su ropa de trabajo por la de chef.

Una vez que el récord fue confirmado por el representante de Guinness, Jack Brockbank, se cantó "Od Yavoh Shalom Aleynu (Salam)" ("La paz nos sea concedida, salam”), que incluye la palabra árabe salam (paz), de modo que la ejecución fue bilingüe hasta cierto punto. Mientras se escuchaba el canto, fueron lanzados al azul del cielo unos globos azules y blancos (los colores de la bandera israelí, hasta ese momento ausentes de la celebración), para satisfacción y aplauso de los jubilosos espectadores judíos y palestinos.

Rafi acudió al representante de Guinness y le preguntó si sabía que el hummus era de fabricación comercial. Rápidamente 
éste respondió: "por supuesto que lo sé, pero en Líbano fue lo mismo... nadie aseveró que el bummus fuera hecho en casa".

De regreso a casa no pude sino reflexionar en la ironía del suceso: un palestino con ciudadanía israelí se propone, en sus propias palabras, "salvar el honor nacional" cocinando el platillo más grande de una comida de su propia herencia étnica, la que recientemente se volvió objeto de competencia de identidad entre árabes y judíos. Lo hace en una villa cuya identidad palestino-musulmana está velada a propósito por razones comerciales; un objetivo logrado por el hummus industrial producido por una compañía judía.

Pero lo más perturbador fue la metáfora en sí misma: Ibrahim Jawadat sugirió que la paz en Medio Oriente se negocia mejor ante un platillo de bummus en el escenario liminal de una villa palestino-israelí. El bummus, sin embargo, olía a podrido, y reflejaba con fidelidad, aunque sin intención, el estado de la paz en la región: inmundo, pútrido y poco apetitoso.

\section{Discusión: el hummus y la solución del conflicto}

En su libro sobre las dimensiones sociales y políticas de la cocina árabe en Israel, la socióloga Liora Gvion ${ }^{37}$ describe cómo la mujer palestina-israelí recoge hierbas silvestres, compra sobras y añade fibras de almidón, con mínimas cantidades de carne y mucho trabajo duro, para alimentar adecuadamente a su familia, pese a su marginación económica y social. Gvion sostiene que estas estrategias culinarias son percibidas por esas mujeres palestinas y sus familiares como estrategias de negociación y subversión e incluso como pequeñas victorias sobre el Estado de Israel y la mayoría judía.

Gvion no se refiere al hummus directamente, acaso porque su tema es la cocina doméstica, mientras que los palestino-israelíes acostumbran comprar el bummus en tiendas o en supermercados propiedad de palestinos. Sin embargo, el platillo pertenece claramente al conjunto de "alimentos de resistencia" palestina que Gvion describe: barato, accesible y muy nutritivo;

${ }^{37}$ L. Gvion, At Stomach Level: Social and Political Aspects of Arab Cuisine in Israel, Jerusalén, Carmel Press, 2006. 
por lo tanto, contribuye a la sobrevivencia y fortaleza de los palestino-israelíes, pues los ayuda a enfrentar las dificultades de pertenecer a una minoría étnica percibida como enemiga por la mayoría de los miembros de la mayoría. El hummus es también percibido por los miembros de este grupo como palestino, o al menos como árabe, lo que lo convierte en un vehículo de su identidad étnica y nacional.

La historiadora Dafna Hirsch ${ }^{38}$ explora las relaciones de los judío-israelíes con el hummus. Sostiene que la comida palestina, en la que el bummus es prominente, fue adoptada por inmigrantes judíos de Europa oriental como parte de su intento por mezclarse con el Medio Oriente desde principios del establecimiento del sionismo a fines del siglo XIX. El conflicto judío-palestino resultante, las guerras entre Israel y sus vecinos árabes y la inmigración de grandes cantidades de judíos de países musulmanes (llamados mizrabi u "orientales") contribuyeron posteriormente a la popularidad del bummus entre los judíoisraelíes, pero eso también llevó a muchos a negar el origen palestino del platillo, arguyendo que era bíblico o que había sido introducido por judíos mizrabi. La producción industrial de bummus en modernas fábricas kosher judías fue otro factor de la absorción material y simbólica de este platillo por el repertorio culinario judío-israelí moderno y de la negación o minusvalía de sus raíces palestinas.

Sin embargo, Hirsch decidió titular su artículo: "El bummus es mejor fresco y preparado por árabes”, aseveración de uno de sus informantes. Ella argumenta que, en años recientes, a medida que el bummus pasa por procesos de gourmetización en Israel y más allá, la identidad árabe de los productores de bummus se vuelve un marcador de autenticidad y, por lo tanto, deviene en activo que asegura la calidad del producto. Hirsch concluye que la percepción judío-israelí del hummus es dinámica e involucra medidas cambiantes del reconocimiento de sus raíces palestinas.

Debe notarse, sin embargo, que el proceso de gourmetización descrito por Hirsch involucra principalmente a miembros de la élite israelí global, quienes están más interesados en las di-

${ }^{38}$ Hirsch, "Hummus is Best when it is Fresh...", op. cit. 
mensiones cosmopolitas del "bummus [gourmet]... preparado por árabes" como medio de distinción social, de expresión de refinamiento y de sofisticación. Pero la maniobra de Jawadat era otra: él se involucró con el bummus como un intento de integrarse a la sociedad judío-israelí, por lo menos hasta cierto punto.

El sociólogo Rafi Grosglik, ${ }^{39}$ en su estudio sobre los alimentos orgánicos en Israel, explora la posición judío-israelí frente al bummus con diferente perspectiva. Argumenta que los productores y consumidores judío-israelíes de bummus orgánico sólo se preocupan por los aspectos del producto relativos a la salud, e ignoran por completo las nociones de sustentabilidad y justicia social, que son parte de la ideología orgánica. Así, mientras Hirsch muestra cómo algunos judío-israelíes reconocen el bummus como palestino, Grosglik sugiere que, aun cuando ese reconocimiento exista, los judío-israelíes de algún modo neutralizan la cuestión problemática de la "identidad nacional" del platillo. Grosglik señala también que el símbolo del hummus está saturado de paradojas y que se caracteriza por su disonancia ideológica.

Mientras Gvion, Hirsch y Grosglik discuten las percepciones del bummus por palestino-israelíes y judío-israelíes, el suceso de Abu Gosh complica el análisis, en la medida en que involucra interacción directa, frente a frente, entre los dos lados de esta dicotomía política. Más aún, la estructura de poder de la hegemonía israelí también fue desafiada en Abu Gosh. Las relaciones de poder cotidianas en Israel involucran una supremacía judía abrumadora. Incluso cuando visitan Abu Gosh para comer en sus restaurantes, los judíos son los clientes ricos que deben ser satisfechos: aquí los palestinos fueron los anfitriones generosos, los propietarios de un capital económico y cultural, en lucha por "restaurar el honor nacional" con una proeza que los judíos no podrían realizar. En este sentido, la propuesta de Ibrahim Jawadat de relajar las tensiones judío-árabes frente a un plato de bummus quedó reafirmada: los palestinos fueron los anfitriones generosos y los judíos se comportaron como debían: humildes, agradecidos y solidarios.

${ }^{39}$ Grosglik, “Organic Hummus in Israel”, op. cit. 
Lo que encontré más intrigante fue el papel de mediadores atribuido por Jawadat a los palestino-israelíes. La aceptación judío-israelí de miembros de este grupo va desde su conceptualización como ciudadanos primitivos de segunda clase, cuya lealtad y derechos son siempre cuestionados, hasta su definición como quinta columna, miembros del campo enemigo que deben ser deportados o, al menos, despojados de su ciudadanía y derechos civiles. Los científicos sociales y los comentaristas políticos arguyen que en las últimas dos décadas los palestinoisraelíes han pasado por un proceso de radicalización religiosa (musulmana) y nacionalista (palestina o árabe) que los aleja del Estado-nación israelí. Paradójicamente, los palestino-israelíes son percibidos a menudo como traidores y colaboradores por la mayoría de los árabes del Medio Oriente, incluso por muchos palestinos no israelíes.

Jawadat, sin embargo, rechazó estas nociones y dio un nuevo significado a la posición liminal de los palestino-israelíes. En vez de aceptar el estatus marginal de ni-uno-ni-otro arriba descrito, sugirió que ellos son ambas cosas, israelíes y palestinos, y que, por lo tanto, pueden servir como puente entre ambos lados. También sugirió que cuando los dos lados se reúnan en el puente deberán discutir sus relaciones y negociar la paz frente a un plato de bummus. De esta manera definió el bummus también como una sustancia mediadora que cierra las brechas (o llena las fisuras, podría decirse) entre judíos y árabes. Aquí convergen la política y la cocina: el bummus fue construido durante el récord Guinness en Abu Gosh, no sólo como receptáculo de identidad israelí o palestina, sino también como un puente entre las partes en conflicto.

La cuestión remanente es “¿por qué el hummus?”. ¿Qué singulariza al alimento en general y al bummus específicamente como medios apropiados para la expresión y negociación de ideas complicadas e incluso de visiones contrastantes? A fin de responder a estas cuestiones debemos regresar a algunos de los atributos básicos de los alimentos como artefactos culturales.

Primero, la naturaleza dúctil y dinámica de los alimentos los hace un vehículo perfecto para ideas complicadas y polisémicas. A diferencia de la mayoría de los artefactos materiales, 
la comida es una materia en cambio constante y rápido. ${ }^{40}$ Lo "crudo" es transformado en lo "cocido" o lo "podrido", y lo cocinado es comido (digerido y defecado) o descartado (antes o después de podrido) en unas cuantas horas. Los alimentos también son menos prescritos y canonizados que la mayoría de los elementos culturales, y siempre presentan grandes variaciones personales, locales y regionales. "El sazón según el gusto" es, por lo tanto, la instrucción de las recetas más meticulosas, lo que subraya el hecho de que la variedad es prácticamente infinita. Esta esencia de cualidades flexibles, cambio constante y gran variedad permite un dinamismo excepcional cuando se trata de significados simbólicos: es la ductilidad de medios la que permite la ductilidad de significados.

Segundo, el hummus tiene en sí mismo características específicas que amplían su capacidad de expresar e incluir contradicciones. Aunque es un platillo mundano, no festivo y barato, hay cientos de recetas, con variaciones y secretos para cocinarlo. Hirsch ${ }^{41}$ arguye que encontrar "el mejor bummus" es casi una obsesión en Israel, lo cual ha sido recientemente reconocido y formalizado por una encuesta nacional (comercialmente orientada) sobre el mejor restaurante de hummus en el país. El hecho de que este platillo tenga tantas variaciones permite la expresión de numerosas ideas sobre diferentes facetas de la identidad nacional y de otro tipo.

El bummus es también un epítome de la comensalidad. La manera más común de comerlo es en un tazón compartido, en el cual se sumergen trozos de pan pita. Aunque puede comerse individualmente, palestinos y judíos rutinariamente hacen a un lado las tendencias individualistas cuando se trata de bummus y lo comparten entusiastas con los comensales, sumergiendo trozos de pita a medio morder en el tazón común. Éste es, por supuesto, un momento íntimo. Uno comparte bummus con parientes y amigos, no con extraños. La invitación a comer bummus es un signo de hospitalidad, amistad y familiaridad. Mi argumento final entonces es que la afirmación de Jawadat de que

${ }^{40}$ C. Levi-Strauss, “The Culinary Triangle”, Partisan Review, vol. 33, otoño de 1966, pp. 586-595; D. Clark, “The Raw and the Rotten: Punk Cuisine”, Ethnology, vol. 43, 2004, pp. 19-31.

${ }^{41}$ Hirsch, "Hummus is Best when it is Fresh...", op. cit. 
compartir bummus es un primer paso hacia la paz en el Medio Oriente no fue sólo una metáfora poética, sino una invitación práctica a iniciar un proceso cultural que puede convertir a los adversarios en amigos.

\section{Traducción del inglés: Ramón Cota Meza}

Dirección institucional del autor:

Department of Sociology and Anthropology

Ben Gurion University of the Negev

POB 653

Beersheba, Israel 84105

avieli@bgu.ac.il

\section{Bibliografía}

ADLER, Ilanit, "Watch: The World Largest Jerusalem Mix”, Channel 2 Neres, 30 de noviembre de 2009. [www.mako.co.il/news-israel/ local/Article-bbeb277c6e54521004.htm, consultado el 4 de marzo de 2015.]

Almog, O., Ha-tzabar-dyokan, Tel Aviv, Am Oved, 1997.

ArIEL, A., "The Hummus Wars", Gastronomica, vol. 12, núm. 1, 2012, pp. 34-42.

Ashrenazi, M. y J. Jacob, The Essence of Japanese Cuisine: An Essay on Food and Culture, Richmond, Curzon, 2000.

Avieli, N., "Roasted Pigs and Bao Dumplings: Festive Food and Imagined Transnational Identity in Chinese-Vietnamese Festivals", Asia Pacific Viewpoint, vol. 46, núm. 3, 2005, pp. 281293.

Avieli, N., "Vietnamese New Year Rice Cakes: Iconic Festive Dishes and Contested National Identity", Ethnology, vol. 44, núm. 2, 2005, pp. 167-187.

BaHLOUl, J., "Food Practices among Sephardic Immigrants in Contemporary France: Dietary Laws in Urban Society", Journal of the American Academy of Religion, vol. 63, núm. 3, 1995, pp. 485496.

Ben-ZE'Ev, E., Remembering Palestine in 1948: Beyond National Narratives, Cambridge, Cambridge University Press, 2011.

Bourdieu, P., Distinction: A Social Critique of the Judgment of Taste, Cambridge, Harvard University Press, 1984. 
Caldwell, M., "Domesticating the French Fry: McDonald's and Consumerism in Moscow", Journal of Consumer Culture, vol. 4, núm. 1, 2004, pp. 5-26.

Cavanaugh, J., "Making Salami, producing Bergamo: The Transformation of Value", Ethnos, vol. 72, núm. 2, 2007, pp. 149-172.

Clark, D., "The Raw and the Rotten: Punk Cuisine", Ethnology, vol. 43, 2004, pp. 19-31.

Counihan, C. M., Around the Tuscan Table: Food, Family and Gender in Twentieth-Century Florence, Nueva York, Routledge, 2004.

Cusack, I., "African Cuisines: Recipes for Nation Building?”, Journal of African Cultural Studies, vol. 13, núm. 2, 2000, pp. 207-225.

CwiertKa, K. J., Modern Japanese Cuisine: Food, Power and National Identity, Londres, Reaktion Books, 2006.

DeSoucey, M., "Gastronationalism Food Traditions and Authenticity Politics in the European Union", American Sociological Review, vol. 75, núm. 3, 2010, pp. 432-455.

Douglas, M., Implicit Meanings: Selected Essays in Anthropology, Hove, Psychology Press, 1975.

Edge, John T., "Hummus catches on in America (as Long as It's Flavored)", The New York Times, 15 de junio de 2010. [www. nytimes.com/2010/06/16/dining/16united.html?_r=0, consultado el 11 de abril de 2013.]

EICHFELD, I., "The 2nd Hummus War”, Yediot Abronot, 28 de enero de 2010. [www.ynetnews.com/articles/0,7340,L-3839025,00. html, consultado en julio de 2013.]

EICHNER, Itamar, "The 2nd Hummus War", Ynetneres, 28 de enero de 2010. [www.ynetnews.com/articles/0,7340,L-3839025,00.html, consultado el 11 de abril de 2013.]

FISCHLER, C., "Food, Self and Identity", Social Science Information/ sur les sciences sociales, vol. 27, núm. 2, 1988, pp. 275-292.

GaLILI, Shuki, "The Third Lebanon War: The Fight over Hummus", Yediot Abronot, 2 de noviembre de 2009. [www.ynet.co.il/ articles/0,7340,L-3798944,00.html, consultado el 4 de marzo de 2015.]

Goldstin, Tani, "Jawadath Ibrahim: Abu Gosh is not only Hummus", Yediot Abronot, 20 de enero de 2006. [www.ynet.co.il/ articles/1,7340,L-3202839,00.html, consultado el 4 de marzo de 2015.]

Goody, J., Cooking, Cuisine and Class: A Study in Comparative Sociology, Cambridge, Cambridge University Press, 1985.

GrosGLIK, R., "Organic Hummus in Israel: Global and Local Ingredients and Images”, Sociological Research Online, vol. 16, núm. 3, 
2011. [www.socresonline.org.uk/16/2/3.html, consultado en julio de 2013.]

Gvion, L., At Stomach Level: Social and Political Aspects of Arab Cuisine in Israel, Jerusalén, Carmel Press, 2006.

Harari, Didi, "Owner of Abu Gosh Restaurant to break the Lebanese Guinness record" (entrevista con Ibrahim Jawadat), 103FM, 8 de noviembre de 2009. [www.103.fm/programs/Media.aspx ?ZrqvnVq=ELLMGD\&c41t4nzVQ=EG, consultado el 4 de marzo de 2015.]

Hirsch, D., "Hummus is Best when it is Fresh and made by Arabs: The Gourmetization of Hummus in Israel and the Return of the Repressed Arab”, American Ethnologist, vol. 38, núm. 4, 2011, pp. 617-630.

Holt, G. y V. Amilien, "Introduction: From Local Food to Localized Food", Anthropology of Food, número especial sobre productos y sistemas agroalimentarios locales, 2007. [aof.revues.org/index405.html, consultado en julio de 2013.]

Howell, S., "Modernizing Mansaf: The Consuming Contexts of Jordan's National Dish", Food and Foodways, vol. 11, núm. 4, 2003, pp. 215-243.

Klumbytè, N., "The Soviet Sausage Renaissance”, American Anthropologist, vol. 112, núm. 1, 2010, pp. 22-37.

Levi-Strauss, C., “The Culinary Triangle”, Partisan Review, vol. 33, otoño de 1966, pp. 586-595.

LichFiELD, G., "Food Fight: A Look Inside the Middle East's New Weapons of Mass Consumption", Foreign Policy, 15 de enero de 2010. [www.foreignpolicy.com/articles/2010/01/15/food_fight, consultado en julio de 2013.]

Local Councils in Israel, "Profile of Local Councils 2009", publicación 1451. [www.cbs.gov.il/publications11/local_authorities09/ pdf/348_0472.pdf, consultado el 4 de marzo de 2015.]

Mennell, S., All Manners of Food: Eating and Taste in England and France from the Middle Ages to the Present, Oxford, Blackwell, 1985.

Mintz, S., Sweetness and Power: The Place of Sugar in Modern History, Nueva York, Viking, 1986.

Narayan, U., Dislocating Cultures: Identities, Traditions and ThirdWorld Feminism, Nueva York-Londres, Routledge, 1997.

O'Connor, K., "The Hawaiian Luau: Food as Tradition, Transgression, Transformation and Travel”, Food, Culture and Society, vol. 11, núm. 2, 2008, pp. 149-172.

Occupied Jerusalem (AFP), "Israelis Seek World Record With 'Jeru- 
salem Mix'”, Al Arabiya Neres, 30 de noviembre de 2009. [www. alarabiya.net/articles/2009/11/30/92799.html, consultado el 4 de marzo de 2015.]

PILCher, Jeffrey M., ;Que vivan los tamales!: Food and the Making of Mexican Identity, Albuquerque, University of New Mexico Press, 1998.

Ries, N., "Potato Ontology: Surviving Postsocialism in Russia", Cultural Anthropology, vol. 24, núm. 2, 2009, pp. 181-212.

SteIn, R., Itineraries of Conflict; Israeli, Palestinians and the Political Lives of Tourism, Durham, Duke University Press, 2003.

SutTon, D. E., Remembrance of Repasts: An Anthropology of Food and Memory, Oxford, Berg, 2001.

Walla Editors, "Another Victory for Israel: Jerusalem Mix Record", Walla! Neres, 30 de noviembre de 2009. [news.walla. co.il/?w $=/ / 1616594$, consultado el 4 de marzo de 2015.]

Watson, J. L. (ed.), Golden Arches East: McDonald's in East Asia, Stanford, Stanford University Press, 1997.

Welz, G., "Contested Origins: Food Heritage and the European Union's Quality Label Program”, Food, Culture and Society, vol. 16, núm. 2, 2013, pp. 265-279.

WILK, R., Home Cooking in the Global Village: Caribbean Food from Buccaneers to Ecotourists, Londres, Berg, 2006.

WILK, R., "'Real Belizean Food': Building Local Identity in the Transnational Caribbean", American Antbropologist, vol. 101, núm. 2, 1999, pp. 244-255.

WINTER, M., "Embeddedness, the New Food Economy and Defensive Localism”, Journal of Rural Studies, vol. 19, núm. 1, 2003, pp. 23-32. 
